\title{
Prevalence and Prognostic Significance of Negative U-waves in a 12-lead Electrocardiogram in the General Population
}

\section{Holkeri, Arttu}

2019-01-15

Holkeri , A, Eranti , A , Haukilahti , M A , Kerola , T , Kenttä , T , Noponen , K, Seppänen , T , Rissanen , H , Heliövaara , M , Knekt , P , Junttila , M J , Huikuri , H V \& Aro , A L 2019 , ' Prevalence and Prognostic Significance of Negative U-waves in a 12-lead Electrocardiogram in the General Population ' , American Journal of Cardiology , vol. 123 , no. 2 , pp. 267-273 . https://doi.org/10.1016/]

http://hdl.handle.net/10138/315637

https://doi.org/10.1016/j.amjcard.2018.10.010

publishedVersion

Downloaded from Helda, University of Helsinki institutional repository.

This is an electronic reprint of the original article.

This reprint may differ from the original in pagination and typographic detail.

Please cite the original version. 


\title{
Prevalence and Prognostic Significance of Negative U-waves in a 12-lead Electrocardiogram in the General Population
}

\author{
Arttu Holkeri, MD ${ }^{\mathrm{a}} *$, Antti Eranti, MD ${ }^{\mathrm{b}}$, M. Anette Haukilahti, BM ${ }^{\mathrm{c}}$, Tuomas Kerola, MD ${ }^{\mathrm{b}}$, \\ Tuomas V. Kenttä, $\mathrm{PhD}^{\mathrm{c}}$, Kai Noponen, $\mathrm{MSc}^{\mathrm{d}}$, Tapio Seppänen, $\mathrm{PhD}^{\mathrm{d}}$, Harri Rissanen, $\mathrm{MSc}^{\mathrm{e}}$, \\ Markku Heliövaara, MD ${ }^{\mathrm{e}}$, Paul Knekt, PHD ${ }^{\mathrm{e}}$, M. Juhani Junttila, MD ${ }^{\mathrm{c}}$, \\ Heikki V. Huikuri, $\mathrm{MD}^{\mathrm{c}}$, and Aapo L. Aro, $\mathrm{MD}^{\mathrm{a}}$
}

\begin{abstract}
Negative U-waves are a relatively rare finding in an electrocardiogram (ECG), but are often associated with cardiac disease. The prognostic significance of negative $U$-waves in the general population is unknown. We evaluated 12-lead ECGs of 6,518 adults $(45 \%$ male, mean age $50.9 \pm 13.8$ years) for the presence of $U$-waves, and followed the subjects for $24.5 \pm 10.3$ years. Primary end points were all-cause mortality, cardiac mortality, and sudden cardiac death; secondary end point was hospitalization due to cardiac causes. Negative $U$-waves (amplitude $\geq \mathbf{0 . 0 5} \mathbf{~ m V}$ ) were present in 231 subjects (3.5\%), minor negative (amplitude $<0.05 \mathrm{mV}$ ) or discordant $\mathrm{U}$-waves in 1,004 subjects $(15.4 \%)$, normal positive $\mathrm{U}$-waves in $3,950(60.6 \%)$ subjects, and no $\mathrm{U}$-waves were observed in 603 subjects $(9.3 \%)$. In 730 subjects $(11.2 \%)$, $U$-waves were unassessable. When adjusted for age and gender, negative $U$-waves were associated with all end points $(p<0.01)$. In an analysis adjusted for multiple demographic and clinical factors, in men, negative $U$-waves were associated with increased risk of all-cause mortality (hazard ratio [HR] 1.60; 95\% confidence interval [CI] 1.26 to 2.03 ; p < 0.001 ), cardiac mortality (HR 1.74; 95\% CI 1.26 to 2.39; $p=0.001$ ), and cardiac hospitalization (HR 1.67; 95\% CI 1.27 to 2.18; p < 0.001), but not with sudden cardiac death, whereas women did not show a significant association to any of the end points $(p>0.30)$. In conclusion, negative $U$-waves are associated with adverse events in the general population. In men, this association is independent of cardiovascular risk factors. ๑) 2018 Elsevier Inc. All rights reserved. (Am J Cardiol 2019;123:267-273)
\end{abstract}

Although first described over 100 years ago, ${ }^{1}$ the $\mathrm{U}$-wave has received much less attention than other components of the ECG, and the mechanisms underlying the genesis of the U-wave are still not fully understood. ${ }^{2}$ Normally,

\footnotetext{
${ }^{a}$ Division of Cardiology, Heart and Lung Center, Helsinki University Hospital, Helsinki, Finland; 'bepartment of Internal Medicine, PäijätHäme Central Hospital, Lahti, Finland; ' ${ }^{\mathrm{R}}$ esearch Unit of Internal Medicine, Medical Research Center, Oulu University Hospital and University of Oulu, Oulu, Finland; ${ }^{\mathrm{d} C e n t e r}$ for Machine Vision and Signal Analysis, University of Oulu, Oulu, Finland; and ${ }^{\mathrm{e}}$ National Institute for Health and Welfare, Helsinki, Finland. Manuscript received July 30, 2018; revised manuscript received and accepted October 5, 2018.

Funding: This work was supported by Ida Montin Foundation (Kerava, Finland), (AH); Finnish Medical Foundation (Helsinki, Finland), (AH/AE/ ALA); Paavo Nurmi Foundation (Helsinki, Finland), (AE); Onni and Hilja Tuovinen's Foundation (Lahti, Finland), (AE); the Orion Research Foundation (Espoo, Finland), (AE/TVK); the Aarne Koskelo Foundation (Helsinki, Finland), (TVK); the Paulo Foundation (Espoo, Finland), (TVK); the Sigrid Juselius Foundation (Helsinki, Finland), (MJJ/HVH/ALA); Academy of Finland (Helsinki, Finland), grant number 267435, (MJJ/HVH); Finnish Foundation for Cardiovascular Research (Helsinki, Finland), (MJJ/HVH); and the Veritas Foundation (Helsinki, Finland), (AE). Funding sources had no involvement in the study design, in the collection, analysis and interpretation of data, in the writing of the report, or in the decision to submit the article for publication.

See page 272 for disclosure information.

*Corresponding author: Tel: +358505 992 179; fax 0947175600.

E-mail address: arttu.holkeri@gmail.com (A. Holkeri).
}

$\mathrm{U}$-waves are concordant with the preceding $\mathrm{T}$-wave and are best seen in the leads V2-V4. ${ }^{3}$ U-wave's amplitude is inversely proportional to heart rate, and it is visible in more than $90 \%$ of ECGs with heart rate under 60 beats/minute. ${ }^{4}$ However, negative U-waves are a much less frequent phenomenon, ${ }^{5}$ and are often associated with cardiac diseases. ${ }^{6-9}$ The prognostic significance of U-wave morphologies has been studied mostly in specific cardiac patient populations. ${ }^{10}$ However, data are lacking on the prognostic significance of different U-wave manifestations in the general population. In the present study, we investigated the prevalence and prognostic significance of negative U-waves and other U-wave morphologies in a large general population sample with a follow-up of 25 years.

\section{Methods}

The study population consists of participants of the MiniFinland Health Survey which was part of the Social Insurance Institution's Finnish Mobile Clinic Survey. The survey included health interviews and examinations. Detailed study protocol and methods are published elsewhere. ${ }^{11}$ Briefly, a total of 8,000 subjects representing the Finnish population aged over 30 years received invitation to the survey in 1978 to 1980 . Of those, 7,217 participated in health examinations. The health interviews included a detailed questionnaire on known diseases, medications, symptoms, and tobacco 
consumption. Health examinations included measuring of heart rate, blood pressure, body mass index, and serum cholesterol level. In addition, plasma potassium levels were obtained from a subgroup of subjects. A resting paper ECG was recorded in supine position from all subjects with Kone Oy's Olli 308 ECG device with a paper speed of $50 \mathrm{~mm} / \mathrm{s}$ and calibration of $10 \mathrm{~mm} / \mathrm{mV}$. After a few months, an additional ECG was recorded from a subgroup of subjects based on the presence of signs of cardiovascular disease. Assessment of baseline diagnoses and the list of diagnoses included as cardiac disease are described in the Supplementary Material. When the original survey was conducted, no institutional review committees existed and universal practice was that subjects gave their consent by participating in the study.

After exclusion of missing ECGs, a total of 6,969 ECGs were digitized and analyzed, as described previously. ${ }^{12}$ In brief, examiners digitized and digitally measured the ECGs, with concurrently manually assessing the presence, deflection, and amplitude of U-waves in each lead. Subjects $(\mathrm{n}=442)$ with bundle branch block, incomplete bundle branch block, atrial fibrillation or flutter, or with rare pathological ECG findings, and subjects $(n=9)$ with missing data were excluded. The remaining 6,518 subjects underwent classification into 4 groups: (1) normal U-waves (positive $\geq 0.05 \mathrm{mV}$ U-wave in $\geq 1$ leads and no negative U-waves), (2) no U-waves in any leads (or only minor positive $<0.05 \mathrm{mV}$ U-waves), (3) negative U-waves (amplitude $\geq 0.05 \mathrm{mV}$ ) in $\geq 1$ leads, and (4) minor negative U-waves (amplitude $<0.05 \mathrm{mV}$ ) or discordant U-waves (positive U-wave with negative preceding T-wave) in $\geq 1$ leads. The rationale behind the classification and assessment of U-waves is presented in detail in the Supplementary Material.

The follow-up phase continued from the baseline examination until December 31, 2011. Nationwide health registries (Statistics Finland and National Hospital Discharge Register) were the source for the follow-up information. Sudden cardiac deaths (SCD) were identified using the modified CAST-criteria ${ }^{13}$ (detailed description is provided in the Supplementary Material). An autopsy was performed on 1,077 cases (27\% of all deceased), of which 194 were SCD cases ( $48 \%$ of all SCD cases). The primary end points were all-cause mortality, cardiac mortality, and SCD. The secondary end point was hospitalization due to cardiac causes.

The general linear model was used for the comparison of the age- and gender-adjusted mean values for continuous variables, and the prevalence of categorical variables. We used the Cox proportional hazards model to estimate hazard ratios (HR) and their 95\% confidence intervals $(95 \% \mathrm{CI})$ between categories of U-waves. The Kaplan-Meier estimator was used to estimate survival function. The multivariate models were adjusted for age, gender, body mass index, systolic blood pressure, serum cholesterol, smoking, heart rate, diabetes, baseline cardiac disease with or without myocardial infarction, and left ventricular hypertrophy $(\mathrm{LVH})$ according to the Sokolow-Lyon ECG criteria. The statistical significance of effect modification by gender and baseline cardiac disease was tested using the Wald test by entering an interaction term of U-waves and gender, and $\mathrm{U}$-waves and cardiac disease, respectively. $\mathrm{p}$ Value of $<0.05$ was considered to be statistically significant. IBM SPSS version 24 served for statistical analysis.

\section{Results}

Of the 6,518 subjects (mean age $50.9 \pm 13.8,45 \%$ male), 3,950 subjects (60.6\%) had normal U-waves, 231 subjects $(3.5 \%)$ presented with negative U-waves, and 1,004 subjects $(15.4 \%)$ presented with minor negative or discordant U-waves; in 603 subjects $(9.3 \%)$, no U-waves were present. In 730 subjects $(11.2 \%)$, U-waves were not assessable, which was generally due to the fusion of U-wave and P-wave due to sinus tachycardia. Examples of different U-wave morphologies are presented in Figure 1.

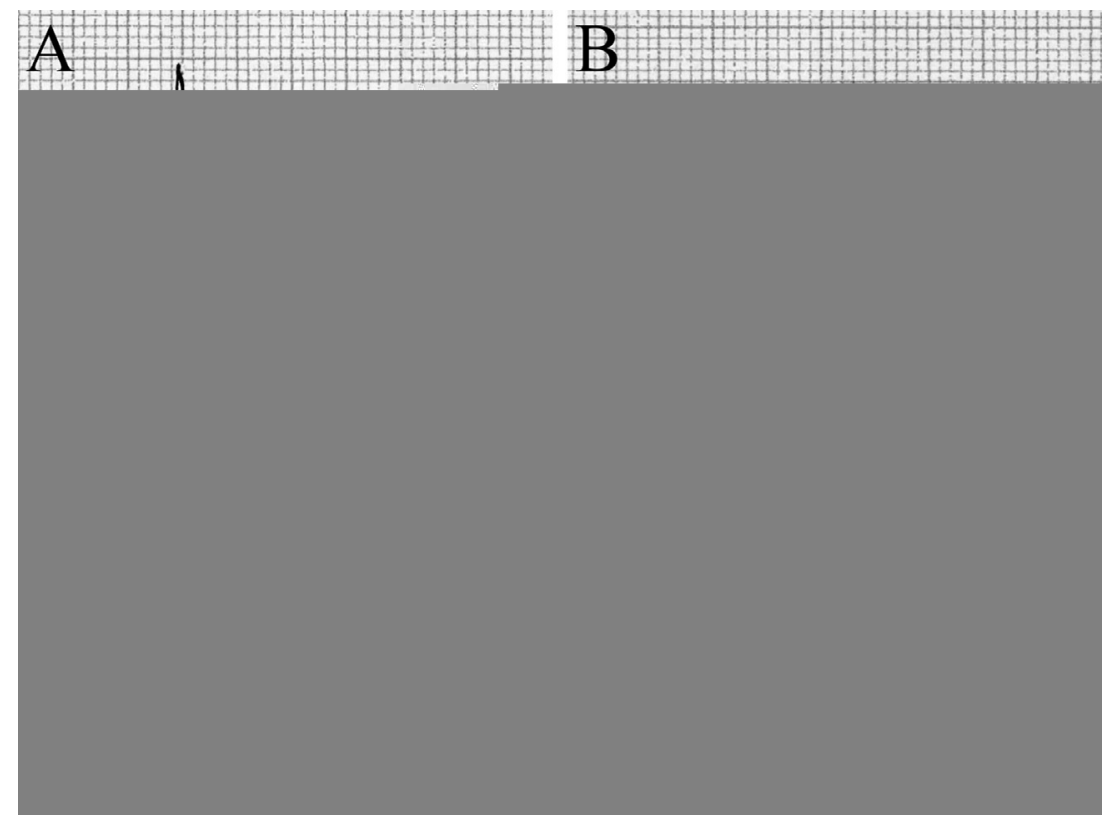

Figure 1. Demonstration different U-wave morphologies. (A) Normal U-wave (amplitude $\geq 0.05 \mathrm{mV}$ ), (B) negative U-wave (amplitude $\geq 0.05 \mathrm{mV}$ ), (C) minor negative $\mathrm{U}$-wave (amplitude $<0.05 \mathrm{mV}$ ), (D) no U-wave. 
Figure 2. Distribution of U-wave morphologies in the 12-lead ECG. Picture A demonstrates the prevalence of U-waves in the ECG. Picture B demonstrates the distribution of U-wave morphologies in leads in which U-waves were present. "Minor negative or disc. U-waves" includes subjects with minor negative U-waves (amplitude $<0.05 \mathrm{mV}$ ) or U-waves discordant with preceding T-wave. "No U-waves" includes subjects with no U-waves or only minor (amplitude $<0.05 \mathrm{mV}$ ) positive $\mathrm{U}$-waves.

The distribution of U-waves in different ECG leads is presented in Figure 2. In $16 \%$ of subjects with negative U-waves, T-wave inversion preceded the negative U-wave.

The baseline characteristics of subjects with different U-wave morphologies are presented in Table 1. Subjects with negative U-waves were older and more likely female, than subjects with normal U-waves ( $p<0.001$ for both). When adjusted for age and gender, subjects with negative U-waves had higher systolic and diastolic blood pressure, higher heart rate, and were more likely to have a history of hypertension, cardiac disease and LVH, compared with subjects with normal U-waves ( $p<0.001$ for all). Overall, $89 \%$ of the subjects with negative U-waves had systolic blood pressure $>140 \mathrm{~mm} \mathrm{Hg}$ or had a cardiac disease.

Serum potassium was obtained from a total of 2,637 subjects (40\%), with a mean level of $4.5 \pm 0.4 \mathrm{mmol} / \mathrm{l}$, with no statistically significant difference between subjects with negative U-waves and normal U-waves. A repeat ECG was recorded after a few months from 78 of the 231 subjects with negative $U$-waves according to the study design. After exclusion of 3 ECGs with unassessable U-waves, a negative U-wave was again observed in $47(63 \%)$ of these subjects. 
Table 1

Baseline characteristics

\begin{tabular}{|c|c|c|c|c|c|}
\hline Variable & $\begin{array}{c}\text { Normal } \\
\text { U-waves } \\
(\mathrm{n}=3,950)\end{array}$ & $\begin{array}{l}\text { Negative } \\
\text { U-waves } \\
(\mathrm{n}=231)\end{array}$ & $\begin{array}{l}\text { Minor negative or } \\
\text { discordant } U \text {-waves } \\
\quad(\mathrm{n}=1,004)\end{array}$ & $\begin{array}{c}\text { No } \\
\text { U-waves } \\
(n=603)\end{array}$ & $\begin{array}{c}\text { Negative U-waves } \\
\text { vs normal U-waves } \\
\text { p value }\end{array}$ \\
\hline Men* & $2,094(53.0 \%)$ & $81(35.1 \%)$ & $354(35.3 \%)$ & $173(28.7 \%)$ & $<0.001$ \\
\hline Systolic blood pressure (mm Hg) & $138.3 \pm 20.1$ & $167.3 \pm 25.9$ & $148.4 \pm 24.6$ & $139.9 \pm 20.4$ & $<0.001$ \\
\hline Diastolic blood pressure $(\mathrm{mm} \mathrm{Hg})$ & $85.3 \pm 11.1$ & $92.8 \pm 12.8$ & $87.8 \pm 11.0$ & $86.9 \pm 10.6$ & $<0.001$ \\
\hline Hypertension diagnose & $460(11.6 \%)$ & $84(36.4 \%)$ & $173(17.2 \%)$ & $99(16.4 \%)$ & $<0.001$ \\
\hline & $265 \pm 50$ & $283 \pm 56$ & $276 \pm 58$ & $269 \pm 53$ & \\
\hline Body mass index $\left(\mathrm{kg} / \mathrm{m}^{2}\right)$ & $25.7 \pm 3.8$ & $25.2 \pm 4.1$ & $25.5 \pm 3.9$ & $26.9 \pm 4.5$ & $<0.001$ \\
\hline Cardiac disease & $557(14.1 \%)$ & $95(41.1 \%)$ & $208(20.7 \%)$ & $104(17.2 \%)$ & $<0.001$ \\
\hline Cardiac disease without myocardial infarction & $455(11.5 \%)$ & $81(35.1 \%)$ & $163(16.2 \%)$ & $77(12.8 \%)$ & $<0.001$ \\
\hline Cardiac disease with myocardial infarction & $102(2.6 \%)$ & $14(6.1 \%)$ & $45(4.5 \%)$ & $27(4.5 \%)$ & 0.69 \\
\hline Diabetes mellitus & $150(3.8 \%)$ & $16(6.9 \%)$ & $45(4.5 \%)$ & $35(5.8 \%)$ & 0.15 \\
\hline
\end{tabular}

In 730 subjects, U-waves were not assessable. Left ventricular hypertrophy according to the Sokolow-Lyon criteria. Diagnoses included as cardiac disease are listed in the Supplementary Material.

* Adjusted for age.

Adjusted for gender.

Adjusted for age and gender.

During the follow-up of $24.5 \pm 10.3$ years, 3,488 subjects $(53.5 \%)$ died, of which 1,509 due to cardiac causes (43.3\% of all deaths), and 358 due to SCD (10.3\% of all deaths). Kaplan-Meier curves for overall mortality according to the U-wave morphology are demonstrated in Figure 3. Table 2 lists the unadjusted and adjusted HRs for all-cause mortality, cardiac death, SCD, and cardiac hospitalization associated with different U-wave morphologies,

Figure 3. Kaplan-Meier survival plots for overall mortality according to the U-wave morphology. "Minor negative or disc. U-waves" stands for minor negative (amplitude $<0.05 \mathrm{mV}$ ) U-waves or U-waves discordant with the preceding T-wave. "Negative U-waves" stands for negative (amplitude $\geq 0.05 \mathrm{mV}$ ) U-waves. "No U-waves" includes subjects with no U-waves or only minor (amplitude $<0.05 \mathrm{mV}$ ) positive U-waves. 
Table 2

Prognostic significance of U-waves

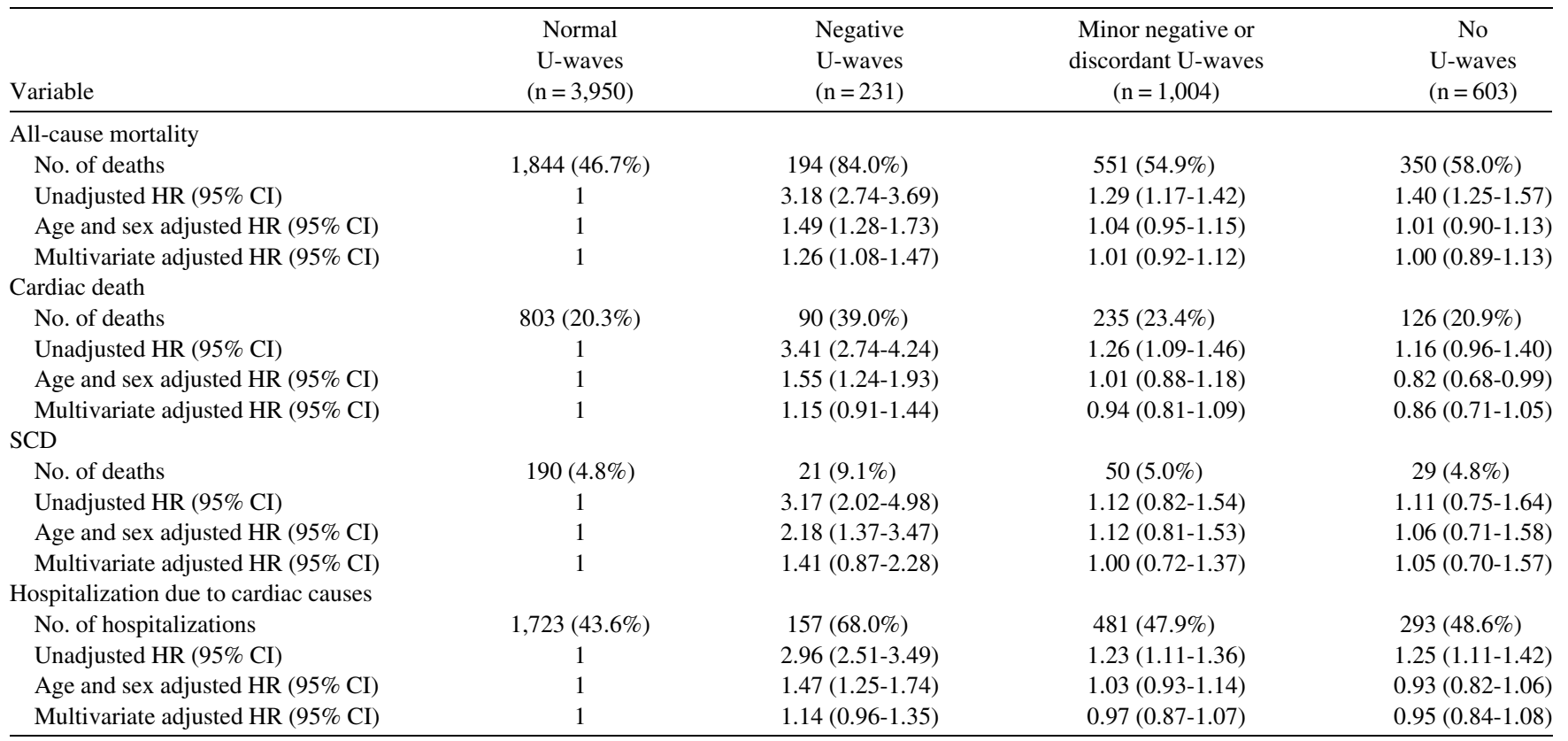

$\mathrm{SCD}=$ sudden cardiac death.

Hazard ratios (HR) and 95\% confidence intervals (CI) were calculated using the Cox proportional hazards model. Variables included in the multivariate analyses were age, gender, systolic blood pressure, heart rate, total serum cholesterol, body mass index, cardiac disease (with or without myocardial infarction), diabetes, left ventricular hypertrophy, and active smoking.

in comparison with normal positive U-waves. In the ageand gender-adjusted analysis, negative U-waves were associated with increased risk for all of the end points, compared with subjects with normal U-waves ( $p<0.01$ for all). In the multivariate analysis, negative $\mathrm{U}$-waves remained independently associated with increased risk for all-cause mortality. Statistically significant effect modification of negative U-waves by baseline cardiac disease was not found on adjusted analyses for all-cause mortality, cardiac death, and cardiac hospitalization.

The baseline characteristics of male and female subjects with negative U-waves were largely similar. After

Table 3

Prognostic significance of negative U-wave in males and females

\begin{tabular}{|c|c|c|c|}
\hline & $\begin{array}{c}\text { Negative U-waves } \\
\text { Male }\end{array}$ & Female & $\mathrm{p}$ Value for sex interaction \\
\hline \multicolumn{4}{|l|}{ Overall mortality } \\
\hline Univariate HR (95\% CI) & $4.89(3.88-6.18)$ & $2.97(2.45-3.61)$ & 0.001 \\
\hline Multivariate HR $(95 \% \mathrm{CI})$ & $1.60(1.26-2.03)$ & $1.09(0.89-1.33)$ & 0.01 \\
\hline \multicolumn{4}{|l|}{ Cardiac death } \\
\hline Univariate HR (95\% CI) & $6.22(4.57-8.47)$ & $2.91(2.13-3.96)$ & 0.001 \\
\hline \multicolumn{4}{|l|}{ SCD } \\
\hline Univariate HR (95\% CI) & $4.94(2.67-9.13)$ & $3.99(2.01-7.92)$ & 0.65 \\
\hline Age adjusted HR (95\% CI) & $2.41(1.29-4.49)$ & $1.94(0.98-3.87)$ & 0.65 \\
\hline Multivariate HR (95\% CI) & $1.51(0.79-2.87)$ & $1.28(0.64-2.58)$ & 0.73 \\
\hline \multicolumn{4}{|c|}{ Hospitalization due to cardiac causes } \\
\hline Univariate HR (95\% CI) & $4.69(3.60-6.11)$ & $2.83(2.29-3.50)$ & 0.003 \\
\hline
\end{tabular}

$\mathrm{SCD}=$ sudden cardiac death.

Hazard ratios (HR) and 95\% confidence intervals (CI) were calculated using the Cox proportional hazards model. Negative U-waves were compared with normal U-waves. Variables included in the multivariate analyses were age, gender, systolic blood pressure, heart rate, total serum cholesterol, body mass index, cardiac disease (with or without myocardial infarction), diabetes, left ventricular hypertrophy, active smoking, and interaction term of U-waves and gender. 
adjusting for age, male and female subjects differed significantly only in heart rate (males $66 \pm 9$ vs females $70 \pm 9 \mathrm{bpm}$ ), serum cholesterol (males $6.7 \pm 1.3 \mathrm{vs}$ females $7.6 \pm 1.4 \mathrm{mmol} / \mathrm{l}$ ), body mass index (males $23.8 \pm 3.4$ vs females $26.0 \pm 4.3 \mathrm{~kg} / \mathrm{m}^{2}$ ), and smoking (males $43.2 \%$ vs females $6.7 \%$; $p<0.01$ for all). No statistically significant differences were found on age, blood pressure, the prevalence of cardiac disease, LVH, or diabetes. Negative U-wave lead distribution did not differ between genders.

The prognostic significance of negative U-waves stratified by gender, and interaction between gender and negative U-waves are presented in Table 3. Significant gender interaction was noted in overall mortality, cardiac mortality, and cardiac hospitalizations. When male and female subjects were analyzed separately, women with negative Uwaves did not show statistically significant increase in the risk of any of the end points in multivariate analysis compared with normal U-waves. On the contrary, in men, negative U-waves were associated with an increase in the risk for all-cause mortality, cardiac death, and hospitalization due to cardiac causes. Furthermore, negative U-waves remained associated with all-cause mortality, cardiac death, and hospitalization due to cardiac causes in a subanalysis of male subjects without cardiac disease (presented in the Supplementary Material). Neither male nor female subjects with negative $\mathrm{U}$-waves showed statistically significant increase in the risk of SCD compared with subjects with normal U-waves.

\section{Discussion}

The present study is the first to directly address the prevalence, characteristics, and prognostic significance of different U-wave morphologies and negative U-waves in the general population. We found that negative $\mathrm{U}$-waves are a relatively rare ECG finding in the general population often associated with older age, female gender, and cardiac diseases. Negative U-waves are in general a marker of poor prognosis; however, in men, they are also independently associated with overall mortality, cardiac death, and cardiac hospitalizations.

Overall, the prevalence and distribution of U-waves and negative U-waves in the present study were similar with earlier reports. ${ }^{3,4,14}$ Our study population had slightly higher prevalence of negative U-waves, 3.5\%, compared with some published reports with prevalences of $1 \%$ to $2 \%, 5,14$ although prevalence as high as $14 \%$ have been reported. ${ }^{15}$

In the present study, subjects with negative U-waves were on average over 15 years older than those with normal U-waves. Similar association of negative U-waves and older age has been demonstrated previously. ${ }^{15,16}$ The presence of negative U-waves is also shown to be a specific marker for cardiac diseases, ${ }^{5,6}$ for example, hypertension $^{9,17}$ and CAD. ${ }^{6,17-19}$ Concordantly, in our study, subjects with negative U-waves had almost $20 \mathrm{~mm} \mathrm{Hg}$ higher systolic blood pressure and were much more likely to have cardiac disease compared with those with normal U-waves.

Although the definite origin of the $\mathrm{U}$-wave has remained unresolved, ${ }^{3}$ according to the prevailing mechanoelectrical hypothesis, U-wave originates from afterpotentials caused by the mechanical stretch during ventricular relaxation. ${ }^{2,20}$ Consequently, myocardium relaxation abnormalities caused by cardiac diseases could be the mechanism behind negative U-waves, ${ }^{21,22}$ which would also explain the adverse prognosis associated with negative $\mathrm{U}$-waves.

The prognostic significance of negative U-waves has been studied only in special patient populations. In hypertensive subjects, negative U-waves have been associated with higher morbidity and mortality, ${ }^{23}$ and in patients with recent myocardial infarction, negative U-waves have been associated with reduced left ventricular ejection fraction, more advanced disease, and increased infarction recurrence rates. ${ }^{10}$ However, the prognostic significance of negative U-waves has not been studied in the general population. In the present study, we demonstrated that negative U-waves in the general population were associated with an increased risk of all-cause mortality. Although much of this risk was explained by traditional cardiovascular risk factors, negative U-waves in the ECGs should prompt investigation of underlying cardiac diseases.

We observed a clear female predominance in subjects with negative U-waves, contrary to previous studies, ${ }^{15,16}$ not explained by blood pressure or the prevalence of cardiac diseases. However, when adjusted for risk factors, negative Uwaves seemed to be a relatively benign finding in women. In contrast, in men, negative U-waves were independently associated with increased mortality, cardiac death, and cardiac hospitalizations. A possible explanation for these gender differences may be different etiologies for negative U-waves in different subpopulations, somewhat similarly to anterior Twave inversions, which are more often observed in women and carry a benign prognosis in this population. ${ }^{24}$ For example, in normal aging, diastolic function decreases more quickly in women compared with men. ${ }^{25}$ As negative Uwaves are hypothesised to result from myocardial relaxation abnormalities, this difference might explain the higher prevalence and better prognosis associated with negative U-waves in women compared with men.

Although the subjects underwent comprehensive health examinations, echocardiography was not performed; consequently, no data were available on the cardiac structure, or on the left ventricular systolic and diastolic function. In addition, coronary heart disease mortality in Finland, especially in the eastern parts of Finland, was one of the highest in the world in the 1960s and 1970s. ${ }^{26}$ As the study population was representative of the Finnish population, with approximately one sixth of the subjects from Eastern Finland, there may be limitations in applying the results of our study to other populations.

In conclusion, negative U-waves are a relatively rare finding in the standard 12-lead ECG in the general population, and are often associated with older age and cardiovascular risk factors and may also be markers of underlying cardiac disease. In addition, negative U-waves are associated with increased cardiac mortality, and especially in men, this association is not fully explained by traditional cardiovascular risk factors, warranting further research.

\section{Disclosures}

None. 


\section{Acknowledgment}

We thank the National Institute for Health and Welfare for their important contribution.

\section{Supplementary materials}

Supplementary material associated with this article can be found, in the online version, at doi:10.1016/j.amj card.2018.10.010.

1. Einthoven W. The different forms of the human electrocardiogram and their signification. Lancet 1912;179:853-861.

2. Eyer K. Support for a mechanico-electrical source of the "U" wave. J Electrocardiol 2015;48:31-32.

3. Perez Riera AR, Ferreira C, Filho CF, Ferreira M, Meneghini A, Uchida AH, Schapachnik E, Dubner S, Zhang L. The enigmatic sixth wave of the electrocardiogram: the U wave. Cardiol J 2008;15: 408-421.

4. Surawicz B. U wave: facts, hypotheses, misconceptions, and misnomers. J Cardiovasc Electrophysiol 1998;9:1117-1128.

5. Fisch C. The clinical electrocardiogram: sensitivity and specificity. ACC Curr J Rev 1997;6:71-75.

6. Kishida H, Cole JS, Surawicz B. Negative U wave: a highly specific but poorly understood sign of heart disease. Am J Cardiol 1982;49:2030-2036.

7. Duke M. Isolated U wave-inversion in acute myocardial infarction. Cardiology 1975;60:220-225.

8. Fisch CY. The electrocardiogram in the early diagnosis of myocardial infarction. ACC Curr J Rev 1995;4:25-28.

9. Twidale N, Gallagher AW, Tonkin AM. Echocardiographic study of U wave inversion in the electrocardiograms of hypertensive patients. J Electrocardiol 1989;22:365-371.

10. Kishida H, Hata N, Nejima J, Suzuki T, Otsu F, Saito T, Kusama Y. Prognosis of patients with persistent negative $\mathrm{U}$ wave following myocardial infarction. Jpn Heart $J$ 1987;28:15-25.

11. Mini-Finland Health Survey - THL. Natl Inst Health Welf THL Finl Available at: http://www.thl.fi/en/web/thlfi-en/research-and-expertwork/population-studies/finnish-mobile-clinic/mini-finland-health-survey. Accessed December 13, 2017.

12. Holkeri A, Eranti A, Kenttä TV, Noponen K, Haukilahti MAE, Seppänen T, Junttila MJ, Kerola T, Rissanen H, Heliövaara M, Knekt $\mathrm{P}$, Aro AL, Huikuri HV. Experiences in digitizing and digitally measuring a paper-based ECG archive. J Electrocardiol 2018;51:74-81.
13. Pratt CM, Greenway PS, Schoenfeld MH, Hibben ML, Reiffel JA. Exploration of the precision of classifying sudden cardiac death. Implications for the interpretation of clinical trials. Circulation 1996;93: 519-524.

14. Palmer JH. Isolated U wave negativity. Circulation 1953;7:205-210.

15. Watanabe N. Clinical significance of the negative $U$ wave in the electrocardiogram. Jpn Circ J 1967;31:9-31.

16. Kishida H, Cole JS, Surawicz B. Negative U wave: the clinical significance and the consideration of pathogenesis. Frontiers of Cardiac Electrophysiology. Springer; 1983. p. 100-119.

17. Gerson MC, McHenry PL. Resting $U$ wave inversion as a marker of stenosis of the left anterior descending coronary artery. Am J Med 1980;69:545-550.

18. Kodama K, Hiasa G, Ohtsuka T, Ikeda S, Hashida H, Kuwahara T, Hara Y, Shigematsu Y, Hamada M, Hiwada K. Transient U wave inversion during treadmill exercise testing in patients with left anterior descending coronary artery disease. Angiology 2000;51:581-589.

19. Chikamori T, Kitaoka H, Matsumura Y, Takata J, Seo H, Doi Y. Clinical and electrocardiographic profiles producing exercise-induced Uwave inversion in patients with severe narrowing of the left anterior descending coronary artery. Am J Cardiol 1997;80:628-632.

20. Schimpf R, Antzelevitch C, Haghi D, Giustetto C, Pizzuti A, Gaita F, Veltmann C, Wolpert C, Borggrefe M. Electromechanical coupling in patients with the short QT syndrome: further insights into the mechanoelectrical hypothesis of the U wave. Heart Rhythm 2008;5:241-245.

21. Bernardo D. di, Murray A. Origin on the electrocardiogram of Uwaves and abnormal U-wave inversion. Cardiovasc Res 2002;53: 202-208.

22. Choo MH, Gibson DG. U waves in ventricular hypertrophy: possible demonstration of mechano-electrical feedback. Br Heart J 1986;55: 428-433.

23. Bellet S, Bettinger JC, Gottlieb H, Kemp RL, Surawicz B. Prognostic significance of negative $\mathrm{U}$ waves in the electrocardiogram in hypertension. Circulation 1957;15:98-101.

24. Aro AL, Anttonen O, Tikkanen JT, Junttila MJ, Kerola T, Rissanen HA, Reunanen A, Huikuri HV. Prevalence and prognostic significance of T-wave inversions in right precordial leads of a 12-lead electrocardiogram in the middle-aged subjects. Circulation 2012;125: 2572-2577.

25. Carvalho J-C, Farand P, Do HD, Brochu M-C, Bonenfant F, Lepage S. Effect of age and sex on echocardiographic left ventricular diastolic function parameters in patients with preserved ejection fraction and normal valvular function. Cardiol J 2013;20:513-518.

26. Jousilahti P, Laatikainen T, Salomaa V, Pietilä A, Vartiainen E, Puska P. 40-year CHD mortality trends and the role of risk factors in mortality decline: the North Karelia project experience. Glob Heart 2016;11: 207-212. 